\title{
CORRECTIONS
}

\section{Increasing the efficiency of D-dimer tests}

The journal on which this Research News story ( $B M J$ 2013;346:f252, doi:10.1136/bmj.f252) was based is the Annals of Internal Medicine [not JAMA Internal Medicine, as published]. We got this wrong in the print version and in the online versions. Additionally, in the print issue and the pdf of the print version, the page numbers were wrong. The correct citation is: Ann Intern Med 2013;158:93-100.

Cite this as: BMJ 2013;346:f542

๑ BMJ Publishing Group Ltd 2013 